\title{
Block Diagonal Matrices
}

\author{
Karol Pąk \\ Institute of Computer Science \\ University of Białystok \\ Poland
}

\begin{abstract}
Summary. In this paper I present basic properties of block diagonal matrices over a set. In my approach the finite sequence of matrices in a block diagonal matrix is not restricted to square matrices. Moreover, the off-diagonal blocks need not be zero matrices, but also with another arbitrary fixed value.
\end{abstract}

MML identifier: MATRIXJ1, version: $\underline{7.9 .01 \quad 4.103 .1019}$

The papers [19], [1], [2], [6], [7], [3], [17], [16], [12], [5], [8], [9], [20], [13], [18], $[21],[4],[14],[15],[11]$, and [10] provide the terminology and notation for this paper.

\section{Preliminaries}

For simplicity, we adopt the following rules: $i, j, m, n, k$ denote natural numbers, $x$ denotes a set, $K$ denotes a field, $a, a_{1}, a_{2}$ denote elements of $K, D$ denotes a non empty set, $d, d_{1}, d_{2}$ denote elements of $D, M, M_{1}, M_{2}$ denote matrices over $D, A, A_{1}, A_{2}, B_{1}, B_{2}$ denote matrices over $K$, and $f, g$ denote finite sequences of elements of $\mathbb{N}$.

One can prove the following propositions:

(1) Let $K$ be a non empty additive loop structure and $f_{1}, f_{2}, g_{1}, g_{2}$ be finite sequences of elements of $K$. If len $f_{1}=\operatorname{len} f_{2}$, then $\left(f_{1}+f_{2}\right)^{\frown}\left(g_{1}+g_{2}\right)=$ $f_{1} \frown g_{1}+f_{2} \frown g_{2}$.

(2) For all finite sequences $f, g$ of elements of $D$ such that $i \in \operatorname{dom} f$ holds $\left(f^{\frown} g\right)_{\lceil i}=\left(f_{\uparrow i}\right)^{\frown} g$.

(3) For all finite sequences $f, g$ of elements of $D$ such that $i \in \operatorname{dom} g$ holds $(f \frown g)_{\uparrow i+\operatorname{len} f}=f \frown\left(g_{\mid i}\right)$. 
(4) If $i \in \operatorname{Seg}(n+1)$, then $((n+1) \mapsto d)_{\mid i}=n \mapsto d$.

(5) $\prod(n \mapsto a)=\operatorname{power}_{K}(a, n)$.

Let us consider $f$ and let $i$ be a natural number. Let us assume that $i \in$ $\operatorname{Seg}\left(\sum f\right)$. The functor $\min (f, i)$ yielding an element of $\mathbb{N}$ is defined by:

(Def. 1) $i \leq \sum f\lceil\min (f, i)$ and $\min (f, i) \in \operatorname{dom} f$ and for every $j$ such that $i \leq \sum f\lceil j$ holds $\min (f, i) \leq j$.

One can prove the following propositions:

(6) If $i \in \operatorname{dom} f$ and $f(i) \neq 0$, then $\min \left(f, \sum f\lceil i)=i\right.$.

(7) If $i \in \operatorname{Seg}\left(\sum f\right)$, then $\min (f, i)-^{\prime} 1=\min (f, i)-1$ and $\sum f \uparrow\left(\min (f, i)-^{\prime}\right.$ 1) $<i$.

(8) If $i \in \operatorname{Seg}\left(\sum f\right)$, then $\min \left(f^{\frown} g, i\right)=\min (f, i)$.

(9) If $i \in \operatorname{Seg}\left(\left(\sum f\right)+\sum g\right) \backslash \operatorname{Seg}\left(\sum f\right)$, then $\min (f \wedge g, i)=\min \left(g, i-{ }^{\prime} \sum f\right)+$ len $f$ and $i-{ }^{\prime} \sum f=i-\sum f$.

(10) If $i \in \operatorname{dom} f$ and $j \in \operatorname{Seg}\left(f_{i}\right)$, then $j+\sum f \uparrow\left(i-{ }^{\prime} 1\right) \in \operatorname{Seg}\left(\sum f\lceil i)\right.$ and $\min \left(f, j+\sum f \uparrow\left(i-{ }^{\prime} 1\right)\right)=i$.

(11) For all $i, j$ such that $i \leq \operatorname{len} f$ and $j \leq \operatorname{len} f$ and $\sum f \nmid i=\sum f \nmid j$ and if $i \in \operatorname{dom} f$, then $f(i) \neq 0$ and if $j \in \operatorname{dom} f$, then $f(j) \neq 0$ holds $i=j$.

\section{Finite Sequences of Matrices}

Let us consider $D$ and let $F$ be a finite sequence of elements of $\left(D^{*}\right)^{*}$. We say that $F$ is matrix-yielding if and only if:

(Def. 2) For every $i$ such that $i \in \operatorname{dom} F$ holds $F(i)$ is a matrix over $D$.

Let us consider $D$. Observe that there exists a finite sequence of elements of $\left(D^{*}\right)^{*}$ which is matrix-yielding.

Let us consider $D$. A finite sequence of matrices over $D$ is a matrix-yielding finite sequence of elements of $\left(D^{*}\right)^{*}$.

Let us consider $K$. A finite sequence of matrices over $K$ is a matrix-yielding finite sequence of elements of $\left((\text { the carrier of } K)^{*}\right)^{*}$.

We now state the proposition

(12) $\emptyset$ is a finite sequence of matrices over $D$.

We adopt the following rules: $F, F_{1}, F_{2}$ are finite sequences of matrices over $D$ and $G, G^{\prime}, G_{1}, G_{2}$ are finite sequences of matrices over $K$.

Let us consider $D, F, x$. Then $F(x)$ is a matrix over $D$.

Let us consider $D, F_{1}, F_{2}$. Then $F_{1} \frown F_{2}$ is a finite sequence of matrices over $D$.

Let us consider $D, M_{1}$. Then $\left\langle M_{1}\right\rangle$ is a finite sequence of matrices over $D$. Let us consider $M_{2}$. Then $\left\langle M_{1}, M_{2}\right\rangle$ is a finite sequence of matrices over $D$. 
Let us consider $D, F, n$. Then $F\lceil n$ is a finite sequence of matrices over $D$. Then $F_{l n}$ is a finite sequence of matrices over $D$.

\section{Sequences of Sizes of Matrices in a Finite Sequence}

Let us consider $D, F$. The functor Len $F$ yielding a finite sequence of elements of $\mathbb{N}$ is defined as follows:

(Def. 3) $\operatorname{dom} \operatorname{Len} F=\operatorname{dom} F$ and for every $i$ such that $i \in \operatorname{dom} L e n F$ holds $($ Len $F)(i)=\operatorname{len} F(i)$.

The functor Width $F$ yields a finite sequence of elements of $\mathbb{N}$ and is defined by:

(Def. 4) $\operatorname{dom}$ Width $F=\operatorname{dom} F$ and for every $i$ such that $i \in \operatorname{dom}$ Width $F$ holds $($ Width $F)(i)=$ width $F(i)$.

Let us consider $D, F$. Then Len $F$ is an element of $\mathbb{N}^{\text {len } F}$. Then Width $F$ is an element of $\mathbb{N}^{\text {len } F}$.

The following propositions are true:

(13) If $\sum$ Len $F=0$, then $\sum$ Width $F=0$.

(14) $\operatorname{Len}\left(F_{1} \frown F_{2}\right)=\left(\operatorname{Len} F_{1}\right) \frown \operatorname{Len} F_{2}$.

(15) $\operatorname{Len}\langle M\rangle=\langle\operatorname{len} M\rangle$.

(16) $\sum \operatorname{Len}\left\langle M_{1}, M_{2}\right\rangle=$ len $M_{1}+$ len $M_{2}$.

(17) $\operatorname{Len}\left(F_{1}\lceil n)=\operatorname{Len} F_{1}\lceil n\right.$.

(18) $\operatorname{Width}\left(F_{1} \frown F_{2}\right)=\left(\text { Width } F_{1}\right)^{\frown}$ Width $F_{2}$.

(19) $\operatorname{Width}\langle M\rangle=\langle$ width $M\rangle$.

(20) $\sum \operatorname{Width}\left\langle M_{1}, M_{2}\right\rangle=$ width $M_{1}+$ width $M_{2}$.

(21) $\operatorname{Width}\left(F_{1}\lceil n)=\right.$ Width $F_{1}\lceil n$.

\section{Block Diagonal Matrices}

Let us consider $D$, let $d$ be an element of $D$, and let $F$ be a finite sequence of matrices over $D$. The $d$-block diagonal of $F$ is a matrix over $D$ and is defined by the conditions (Def. 5).

(Def. 5)(i) len (the $d$-block diagonal of $F$ ) $=\sum$ Len $F$,

(ii) width (the $d$-block diagonal of $F$ ) $=\sum$ Width $F$, and

(iii) for all $i, j$ such that $\langle i, j\rangle \in$ the indices of the $d$ block diagonal of $F$ holds if $j \leq \sum$ Width $F \uparrow\left(\min (\operatorname{Len} F, i)-^{\prime}\right.$ 1) or $j>\sum$ Width $F\lceil\min (\operatorname{Len} F, i)$, then (the $d$-block diagonal of $F)_{i, j}=d$ and if $\sum \operatorname{Width} F \uparrow\left(\min (\operatorname{Len} F, i)-^{\prime} 1\right)<j \leq$ $\sum$ Width $F\lceil\min (\operatorname{Len} F, i) \text {, then (the } d \text {-block diagonal of } F)_{i, j}=$ $F(\min (\operatorname{Len} F, i))_{i-^{\prime}} \sum \operatorname{Len} F \uparrow\left(\min (\operatorname{Len} F, i)-^{\prime} 1\right), j-^{\prime} \sum \operatorname{Width} F \uparrow\left(\min (\operatorname{Len} F, i)-^{\prime} 1\right)$. 
Let us consider $D$, let $d$ be an element of $D$, and let $F$ be a finite sequence of matrices over $D$. Then the $d$-block diagonal of $F$ is a matrix over $D$ of dimension $\sum$ Len $F \times \sum$ Width $F$.

Next we state a number of propositions:

(22) For every finite sequence $F$ of matrices over $D$ such that $F=\emptyset$ holds the $d$-block diagonal of $F=\emptyset$.

(23) Let $M$ be a matrix over $D$ of dimension $\sum \operatorname{Len}\left\langle M_{1}, M_{2}\right\rangle \times \sum \operatorname{Width}\left\langle M_{1}\right.$, $\left.M_{2}\right\rangle$. Then $M=$ the $d$-block diagonal of $\left\langle M_{1}, M_{2}\right\rangle$ if and only if for every $i$ holds if $i \in \operatorname{dom} M_{1}$, then $\operatorname{Line}(M, i)=\operatorname{Line}\left(M_{1}, i\right)^{\frown}\left(\right.$ width $\left.M_{2} \mapsto d\right)$ and if $i \in \operatorname{dom} M_{2}$, then Line $\left(M, i+\operatorname{len} M_{1}\right)=\left(\right.$ width $\left.M_{1} \mapsto d\right){ }^{\frown} \operatorname{Line}\left(M_{2}, i\right)$.

(24) Let $M$ be a matrix over $D$ of dimension $\sum \operatorname{Len}\left\langle M_{1}, M_{2}\right\rangle \times \sum \operatorname{Width}\left\langle M_{1}\right.$, $\left.M_{2}\right\rangle$. Then $M=$ the $d$-block diagonal of $\left\langle M_{1}, M_{2}\right\rangle$ if and only if for every $i$ holds if $i \in \operatorname{Seg}$ width $M_{1}$, then $M_{\square, i}=\left(\left(M_{1}\right)_{\square, i}\right)^{\frown}\left(\operatorname{len} M_{2} \mapsto d\right)$ and if $i \in \operatorname{Seg}$ width $M_{2}$, then $M_{\square, i+\text { width } M_{1}}=\left(\text { len } M_{1} \mapsto d\right)^{\frown}\left(\left(M_{2}\right)_{\square, i}\right)$.

(25) The indices of the $d_{1}$-block diagonal of $F_{1}$ is a subset of the indices of the $d_{2}$-block diagonal of $F_{1} \frown F_{2}$.

(26) Suppose $\langle i, j\rangle \in$ the indices of the $d$-block diagonal of $F_{1}$. Then (the $d$-block diagonal of $\left.F_{1}\right)_{i, j}=\left(\text { the } d \text {-block diagonal of } F_{1} \frown F_{2}\right)_{i, j}$.

(27) $\langle i, j\rangle \in$ the indices of the $d_{1}$-block diagonal of $F_{2}$ if and only if $i>0$ and $j>0$ and $\left\langle i+\sum \operatorname{Len} F_{1}, j+\sum\right.$ Width $\left.F_{1}\right\rangle \in$ the indices of the $d_{2}$-block diagonal of $F_{1} \frown F_{2}$.

(28) Suppose $\langle i, j\rangle \in$ the indices of the $d$-block diagonal of $F_{2}$. Then (the $d$-block diagonal of $\left.F_{2}\right)_{i, j}=$ (the $d$-block diagonal of $F_{1} \frown$ $\left.F_{2}\right)_{i+\sum \text { Len }} F_{1}, j+\sum$ Width $F_{1}$.

(29) Suppose $\langle i, j\rangle \in$ the indices of the $d$-block diagonal of $F_{1} \frown F_{2}$ but $i \leq \sum$ Len $F_{1}$ and $j>\sum$ Width $F_{1}$ or $i>\sum$ Len $F_{1}$ and $j \leq \sum$ Width $F_{1}$. Then (the $d$-block diagonal of $\left.F_{1} \frown F_{2}\right)_{i, j}=d$.

(30) Let given $i, j, k$. Suppose $i \in \operatorname{dom} F$ and $\langle j, k\rangle \in$ the indices of $F(i)$. Then

(i) $\left\langle j+\sum \operatorname{Len} F \uparrow\left(i-{ }^{\prime} 1\right), k+\sum\right.$ Width $\left.F \uparrow\left(i-{ }^{\prime} 1\right)\right\rangle \in$ the indices of the $d$-block diagonal of $F$, and

(ii) $\quad F(i)_{j, k}=(\text { the } d \text {-block diagonal of } F)_{j+\sum \operatorname{Len} F \uparrow\left(i-^{\prime} 1\right), k+\sum \text { Width } F \uparrow\left(i-^{\prime} 1\right)}$.

(31) If $i \in \operatorname{dom} F$, then $F(i)=\operatorname{Segm}($ the $d$-block diagonal of $F, \quad \operatorname{Seg}\left(\sum \operatorname{Len} F\lceil i) \backslash \operatorname{Seg}\left(\sum \operatorname{Len} F \uparrow\left(i{ }^{\prime} \quad 1\right)\right), \operatorname{Seg}\left(\sum\right.\right.$ Width $F\lceil i) \backslash$ $\operatorname{Seg}\left(\sum\right.$ Width $\left.\left.F \uparrow\left(i-^{\prime} 1\right)\right)\right)$.

(32) $\quad M=\operatorname{Segm}($ the $d$-block diagonal of $\langle M\rangle \frown F$, Seg len $M$, Seg width $M$ ).

(33) $\quad M=\operatorname{Segm}\left(\right.$ the $d$-block diagonal of $F \frown\langle M\rangle, \operatorname{Seg}\left(\operatorname{len} M+\sum \operatorname{Len} F\right) \backslash$ $\operatorname{Seg}\left(\sum \operatorname{Len} F\right), \operatorname{Seg}\left(\right.$ width $M+\sum$ Width $\left.F\right) \backslash \operatorname{Seg}\left(\sum\right.$ Width $\left.\left.F\right)\right)$.

(34) The $d$-block diagonal of $\langle M\rangle=M$. 
(35) The $d$-block diagonal of $F_{1} \frown F_{2}=$ the $d$-block diagonal of $\langle$ the $d$-block diagonal of $\left.F_{1}\right\rangle^{\frown} F_{2}$.

(36) The $d$-block diagonal of $F_{1} \frown F_{2}=$ the $d$-block diagonal of $F_{1} \frown\langle$ the $d$-block diagonal of $\left.F_{2}\right\rangle$.

(37) If $i \in \operatorname{Seg}\left(\sum \operatorname{Len} F\right)$ and $m=\min (\operatorname{Len} F, i)$, then Line(the $d$-block diagonal of $F, i)=\left(\left(\sum \operatorname{Width}\left(F \uparrow\left(m-^{\prime} 1\right)\right)\right) \mapsto d\right)^{\frown} \operatorname{Line}\left(F(m), i-^{\prime}\right.$ $\sum \operatorname{Len}\left(F\left\lceil\left(m-^{\prime} 1\right)\right)\right)^{\frown}\left(\left(\left(\sum \operatorname{Width} F\right)-^{\prime} \sum \operatorname{Width}(F\lceil m)) \mapsto d\right)\right.$.

(38) If $i \in \operatorname{Seg}\left(\sum\right.$ Width $\left.F\right)$ and $m=\min ($ Width $F, i)$, then (the $d$-block diagonal of $F)_{\square, i}=\left(\left(\sum \operatorname{Len}\left(F \uparrow\left(m-{ }^{\prime} 1\right)\right)\right) \mapsto d\right)^{\frown}$

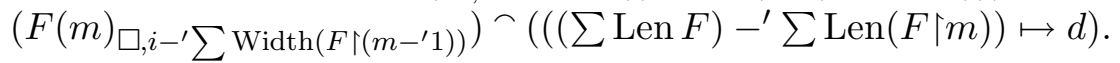

(39) Let $M_{1}, M_{2}, N_{1}, N_{2}$ be matrices over $D$. Suppose len $M_{1}=$ len $N_{1}$ and width $M_{1}=$ width $N_{1}$ and len $M_{2}=\operatorname{len} N_{2}$ and width $M_{2}=$ width $N_{2}$ and the $d_{1}$-block diagonal of $\left\langle M_{1}, M_{2}\right\rangle=$ the $d_{2}$-block diagonal of $\left\langle N_{1}, N_{2}\right\rangle$. Then $M_{1}=N_{1}$ and $M_{2}=N_{2}$.

(40) Suppose $M=\emptyset$. Then

(i) the $d$-block diagonal of $F^{\frown}\langle M\rangle=$ the $d$-block diagonal of $F$, and

(ii) the $d$-block diagonal of $\left\langle M \wedge^{\frown} F=\right.$ the $d$-block diagonal of $F$.

(41) Suppose $i \in \operatorname{dom} A$ and width $A=$ width (the deleting of $i$-row in $A$ ). Then the deleting of $i$-row in the $a$-block diagonal of $\langle A\rangle^{\frown} G=$ the $a$-block diagonal of $\langle\text { the deleting of } i \text {-row in } A\rangle^{\frown} G$.

(42) Suppose $i \in \operatorname{dom} A$ and width $A=$ width (the deleting of $i$-row in $A$ ). Then the deleting of $\left(\sum \operatorname{Len} G\right)+i$-row in the $a$-block diagonal of $G^{\wedge}\langle A\rangle=$ the $a$-block diagonal of $G^{\frown}\langle$ the deleting of $i$-row in $A\rangle$.

(43) Suppose $i \in \operatorname{Seg}$ width $A$. Then the deleting of $i$-column in the $a$-block diagonal of $\langle A\rangle^{\frown} G=$ the $a$-block diagonal of $\langle$ the deleting of $i$-column in A) $\frown$ G.

(44) Suppose $i \in \operatorname{Seg}$ width $A$. Then the deleting of $i+\sum$ Width $G$-column in the $a$-block diagonal of $G^{\frown}\langle A\rangle=$ the $a$-block diagonal of $G^{\frown}\langle$ the deleting of $i$-column in $A\rangle$.

Let us consider $D$ and let $F$ be a finite sequence of elements of $\left(D^{*}\right)^{*}$. We say that $F$ is square-matrix-yielding if and only if:

(Def. 6) For every $i$ such that $i \in \operatorname{dom} F$ there exists $n$ such that $F(i)$ is a square matrix over $D$ of dimension $n$.

Let us consider $D$. One can verify that there exists a finite sequence of elements of $\left(D^{*}\right)^{*}$ which is square-matrix-yielding.

Let us consider $D$. Observe that every finite sequence of elements of $\left(D^{*}\right)^{*}$ which is square-matrix-yielding is also matrix-yielding.

Let us consider $D$. A finite sequence of square-matrices over $D$ is a squarematrix-yielding finite sequence of elements of $\left(D^{*}\right)^{*}$. 
Let us consider $K$. A finite sequence of square-matrices over $K$ is a squarematrix-yielding finite sequence of elements of $\left((\text { the carrier of } K)^{*}\right)^{*}$.

We use the following convention: $S, S_{1}, S_{2}$ denote finite sequences of squarematrices over $D$ and $R, R_{1}, R_{2}$ denote finite sequences of square-matrices over $K$.

One can prove the following proposition

(45) $\emptyset$ is a finite sequence of square-matrices over $D$.

Let us consider $D, S, x$. Then $S(x)$ is a square matrix over $D$ of dimension len $S(x)$.

Let us consider $D, S_{1}, S_{2}$. Then $S_{1} \frown S_{2}$ is a finite sequence of square-matrices over $D$.

Let us consider $D, n$ and let $M_{1}$ be a square matrix over $D$ of dimension $n$. Then $\left\langle M_{1}\right\rangle$ is a finite sequence of square-matrices over $D$.

Let us consider $D, n, m$, let $M_{1}$ be a square matrix over $D$ of dimension $n$, and let $M_{2}$ be a square matrix over $D$ of dimension $m$. Then $\left\langle M_{1}, M_{2}\right\rangle$ is a finite sequence of square-matrices over $D$.

Let us consider $D, S, n$. Then $S\lceil n$ is a finite sequence of square-matrices over $D$. Then $S_{\lfloor n}$ is a finite sequence of square-matrices over $D$.

The following proposition is true

(46) $\quad$ Len $S=$ Width $S$.

Let us consider $D$, let $d$ be an element of $D$, and let $S$ be a finite sequence of square-matrices over $D$. Then the $d$-block diagonal of $S$ is a square matrix over $D$ of dimension $\sum$ Len $S$.

One can prove the following propositions:

(47) Let $A$ be a square matrix over $K$ of dimension $n$. Suppose $i \in \operatorname{dom} A$ and $j \in \operatorname{Seg} n$. Then the deleting of $i$-row and $j$-column in the $a$-block diagonal of $\langle A\rangle^{\wedge} R=$ the $a$-block diagonal of $\langle$ the deleting of $i$-row and $j$-column in $A\rangle\urcorner R$.

(48) Let $A$ be a square matrix over $K$ of dimension $n$. Suppose $i \in \operatorname{dom} A$ and $j \in \operatorname{Seg} n$. Then the deleting of $i+\sum$ Len $R$-row and $j+\sum$ Len $R$-column in the $a$-block diagonal of $R^{\frown}\langle A\rangle=$ the $a$-block diagonal of $\left.R\right\urcorner\langle$ the deleting of $i$-row and $j$-column in $A\rangle$.

Let us consider $K, R$. The functor Det $R$ yielding a finite sequence of elements of $K$ is defined as follows:

(Def. 7) $\operatorname{dom} \operatorname{Det} R=\operatorname{dom} R$ and for every $i$ such that $i \in \operatorname{dom} \operatorname{Det} R$ holds $(\operatorname{Det} R)(i)=\operatorname{Det} R(i)$.

Let us consider $K, R$. Then $\operatorname{Det} R$ is an element of (the carrier of $K)^{\text {len } R}$.

In the sequel $N$ denotes a square matrix over $K$ of dimension $n$ and $N_{1}$ denotes a square matrix over $K$ of dimension $m$.

The following propositions are true: 
(49) $\operatorname{Det}\langle N\rangle=\langle\operatorname{Det} N\rangle$.

(50) $\operatorname{Det}\left(R_{1} \frown R_{2}\right)=\left(\operatorname{Det} R_{1}\right)^{\frown} \operatorname{Det} R_{2}$.

(51) $\operatorname{Det}(R\lceil n)=\operatorname{Det} R\lceil n$.

(52) $\operatorname{Det}\left(\right.$ the $0_{K}$-block diagonal of $\left.\left\langle N, N_{1}\right\rangle\right)=\operatorname{Det} N \cdot \operatorname{Det} N_{1}$.

(53) $\operatorname{Det}\left(\right.$ the $0_{K}$-block diagonal of $R$ ) $=\prod \operatorname{Det} R$.

(54) If len $A_{1} \neq$ width $A_{1}$ and $N=$ the $0_{K}$-block diagonal of $\left\langle A_{1}, A_{2}\right\rangle$, then $\operatorname{Det} N=0_{K}$.

(55) Suppose Len $G \neq$ Width $G$. Let $M$ be a square matrix over $K$ of dimension $n$. If $M=$ the $0_{K}$-block diagonal of $G$, then Det $M=0_{K}$.

\section{An Example of a Finite Sequence of Matrices}

Let us consider $K$ and let $f$ be a finite sequence of elements of $\mathbb{N}$. The functor $I_{K}^{f \times f}$ yielding a finite sequence of square-matrices over $K$ is defined by:

(Def. 8) $\operatorname{dom}\left(I_{K}^{f \times f}\right)=\operatorname{dom} f$ and for every $i$ such that $i \in \operatorname{dom}\left(I_{K}^{f \times f}\right)$ holds $I_{K}^{f \times f}(i)=I_{K}^{f(i) \times f(i)}$.

The following propositions are true:

(56) $\operatorname{Len}\left(I_{K}^{f \times f}\right)=f$ and $\operatorname{Width}\left(I_{K}^{f \times f}\right)=f$.

(57) For every element $i$ of $\mathbb{N}$ holds $I_{K}^{\langle i\rangle \times\langle i\rangle}=\left\langle I_{K}^{i \times i}\right\rangle$.

(58) $I_{K}^{\left(f^{\wedge} g\right) \times\left(f^{\wedge} g\right)}=\left(I_{K}^{f \times f}\right) \frown I_{K}^{g \times g}$.

(59) $\quad I_{K}^{(f\lceil n) \times(f\lceil n)}=I_{K}^{f \times f}\lceil n$.

(60) The $0_{K}$-block diagonal of $\left\langle I_{K}^{i \times i}, I_{K}^{j \times j}\right\rangle=I_{K}^{(i+j) \times(i+j)}$.

(61) The $0_{K}$-block diagonal of $I_{K}^{f \times f}=I_{K}^{\left(\sum f\right) \times\left(\sum f\right)}$.

In the sequel $p, p_{1}$ are finite sequences of elements of $K$.

\section{Operations on a Finite Sequence of Matrices}

Let us consider $K, G, p$. The functor $p \bullet G$ yielding a finite sequence of matrices over $K$ is defined as follows:

(Def. 9) $\operatorname{dom}(p \bullet G)=\operatorname{dom} G$ and for every $i$ such that $i \in \operatorname{dom}(p \bullet G)$ holds $(p \bullet G)(i)=p_{i} \cdot G(i)$.

Let us consider $K$ and let us consider $R, p$. Then $p \bullet R$ is a finite sequence of square-matrices over $K$.

The following propositions are true:

(62) $\operatorname{Len}(p \bullet G)=\operatorname{Len} G$ and $\operatorname{Width}(p \bullet G)=\operatorname{Width} G$.

(63) $p \bullet\langle A\rangle=\left\langle p_{1} \cdot A\right\rangle$.

(64) If len $G=\operatorname{len} p$ and len $G_{1} \leq$ len $p_{1}$, then $p^{\frown} p_{1} \bullet G^{\frown} G_{1}=(p \bullet G)^{\frown}\left(p_{1} \bullet G_{1}\right)$. 
(65) $a \cdot$ the $a_{1}$-block diagonal of $G=$ the $\left(a \cdot a_{1}\right)$-block diagonal of len $G \mapsto a \bullet G$.

Let us consider $K$ and let $G_{1}, G_{2}$ be finite sequences of matrices over $K$. The functor $G_{1} \oplus G_{2}$ yields a finite sequence of matrices over $K$ and is defined by:

(Def. 10) $\operatorname{dom}\left(G_{1} \oplus G_{2}\right)=\operatorname{dom} G_{1}$ and for every $i$ such that $i \in \operatorname{dom}\left(G_{1} \oplus G_{2}\right)$ holds $\left(G_{1} \oplus G_{2}\right)(i)=G_{1}(i)+G_{2}(i)$.

Let us consider $K$ and let us consider $R, G$. Then $R \oplus G$ is a finite sequence of square-matrices over $K$.

The following propositions are true:

(66) $\operatorname{Len}\left(G_{1} \oplus G_{2}\right)=\operatorname{Len} G_{1}$ and $\operatorname{Width}\left(G_{1} \oplus G_{2}\right)=\operatorname{Width} G_{1}$.

(67) If len $G=\operatorname{len} G^{\prime}$, then $G^{\frown} G_{1} \oplus G^{\prime} \frown G_{2}=\left(G \oplus G^{\prime}\right)^{\frown}\left(G_{1} \oplus G_{2}\right)$.

(68) $\langle A\rangle \oplus G=\langle A+G(1)\rangle$.

(69) $\left\langle A_{1}\right\rangle \oplus\left\langle A_{2}\right\rangle=\left\langle A_{1}+A_{2}\right\rangle$.

(70) $\left\langle A_{1}, B_{1}\right\rangle \oplus\left\langle A_{2}, B_{2}\right\rangle=\left\langle A_{1}+A_{2}, B_{1}+B_{2}\right\rangle$.

(71) Suppose len $A_{1}=$ len $B_{1}$ and len $A_{2}=$ len $B_{2}$ and width $A_{1}=$ width $B_{1}$ and width $A_{2}=$ width $B_{2}$. Then (the $a_{1}$-block diagonal of $\left.\left\langle A_{1}, A_{2}\right\rangle\right)+$ (the $a_{2}$-block diagonal of $\left.\left\langle B_{1}, B_{2}\right\rangle\right)=$ the $\left(a_{1}+a_{2}\right)$-block diagonal of $\left\langle A_{1}, A_{2}\right\rangle \oplus$ $\left\langle B_{1}, B_{2}\right\rangle$.

(72) Suppose Len $R_{1}=$ Len $R_{2}$ and Width $R_{1}=$ Width $R_{2}$. Then (the $a_{1^{-}}$ block diagonal of $\left.R_{1}\right)+\left(\right.$ the $a_{2}$-block diagonal of $\left.R_{2}\right)=$ the $\left(a_{1}+a_{2}\right)$-block diagonal of $R_{1} \oplus R_{2}$.

Let us consider $K$ and let $G_{1}, G_{2}$ be finite sequences of matrices over $K$. The functor $G_{1} G_{2}$ yielding a finite sequence of matrices over $K$ is defined by:

(Def. 11) $\operatorname{dom}\left(G_{1} G_{2}\right)=\operatorname{dom} G_{1}$ and for every $i$ such that $i \in \operatorname{dom}\left(G_{1} G_{2}\right)$ holds $\left(G_{1} G_{2}\right)(i)=G_{1}(i) \cdot G_{2}(i)$.

Next we state several propositions:

(73) If Width $G_{1}=\operatorname{Len} G_{2}$, then $\operatorname{Len}\left(G_{1} G_{2}\right)=\operatorname{Len} G_{1}$ and Width $\left(G_{1} G_{2}\right)=$ Width $G_{2}$.

(74) If len $G=\operatorname{len} G^{\prime}$, then $\left(G^{\frown} G_{1}\right)\left(G^{\prime} \frown G_{2}\right)=\left(G G^{\prime}\right) \frown\left(G_{1} G_{2}\right)$.

(75) $\langle A\rangle G=\langle A \cdot G(1)\rangle$.

(76) $\left\langle A_{1}\right\rangle\left\langle A_{2}\right\rangle=\left\langle A_{1} \cdot A_{2}\right\rangle$.

(77) $\left\langle A_{1}, B_{1}\right\rangle\left\langle A_{2}, B_{2}\right\rangle=\left\langle A_{1} \cdot A_{2}, B_{1} \cdot B_{2}\right\rangle$.

(78) Suppose width $A_{1}=\operatorname{len} B_{1}$ and width $A_{2}=\operatorname{len} B_{2}$. Then (the $0_{K}$-block diagonal of $\left.\left\langle A_{1}, A_{2}\right\rangle\right) \cdot\left(\right.$ the $0_{K}$-block diagonal of $\left.\left\langle B_{1}, B_{2}\right\rangle\right)=$ the $0_{K}$-block diagonal of $\left\langle A_{1}, A_{2}\right\rangle\left\langle B_{1}, B_{2}\right\rangle$.

(79) If Width $R_{1}=\operatorname{Len} R_{2}$, then (the $0_{K}$-block diagonal of $R_{1}$ ) (the $0_{K}$-block diagonal of $R_{2}$ ) = the $0_{K}$-block diagonal of $R_{1} R_{2}$. 


\section{REFERENCES}

[1] Grzegorz Bancerek. Cardinal numbers. Formalized Mathematics, 1(2):377-382, 1990.

[2] Grzegorz Bancerek. The fundamental properties of natural numbers. Formalized Mathematics, 1(1):41-46, 1990.

[3] Grzegorz Bancerek and Krzysztof Hryniewiecki. Segments of natural numbers and finite sequences. Formalized Mathematics, 1(1):107-114, 1990.

[4] Czesław Byliński. Binary operations applied to finite sequences. Formalized Mathematics, 1(4):643-649, 1990.

[5] Czesław Byliński. Finite sequences and tuples of elements of a non-empty sets. Formalized Mathematics, 1(3):529-536, 1990.

[6] Czesław Byliński. Functions and their basic properties. Formalized Mathematics, 1(1):5565, 1990.

[7] Czesław Byliński. Partial functions. Formalized Mathematics, 1(2):357-367, 1990.

[8] Katarzyna Jankowska. Matrices. Abelian group of matrices. Formalized Mathematics, $2(4): 475-480,1991$

[9] Katarzyna Jankowska. Transpose matrices and groups of permutations. Formalized Mathematics, 2(5):711-717, 1991.

[10] Andrzej Kondracki. The Chinese Remainder Theorem. Formalized Mathematics, 6(4):573-577, 1997.

[11] Jarosław Kotowicz. Functions and finite sequences of real numbers. Formalized Mathematics, 3(2):275-278, 1992.

[12] Eugeniusz Kusak, Wojciech Leończuk, and Michał Muzalewski. Abelian groups, fields and vector spaces. Formalized Mathematics, 1(2):335-342, 1990.

[13] Takaya Nishiyama and Yasuho Mizuhara. Binary arithmetics. Formalized Mathematics, 4(1):83-86, 1993.

[14] Karol Pąk. Basic properties of the rank of matrices over a field. Formalized Mathematics, 15(4):199-211, 2007.

[15] Karol Pạk and Andrzej Trybulec. Laplace expansion. Formalized Mathematics, 15(3):143$150,2007$.

[16] Wojciech A. Trybulec. Groups. Formalized Mathematics, 1(5):821-827, 1990.

[17] Wojciech A. Trybulec. Vectors in real linear space. Formalized Mathematics, 1(2):291-296, 1990.

[18] Wojciech A. Trybulec. Lattice of subgroups of a group. Frattini subgroup. Formalized Mathematics, 2(1):41-47, 1991.

[19] Zinaida Trybulec. Properties of subsets. Formalized Mathematics, 1(1):67-71, 1990.

[20] Katarzyna Zawadzka. The sum and product of finite sequences of elements of a field. Formalized Mathematics, 3(2):205-211, 1992.

[21] Katarzyna Zawadzka. The product and the determinant of matrices with entries in a field. Formalized Mathematics, 4(1):1-8, 1993.

Received May 13, 2008 